
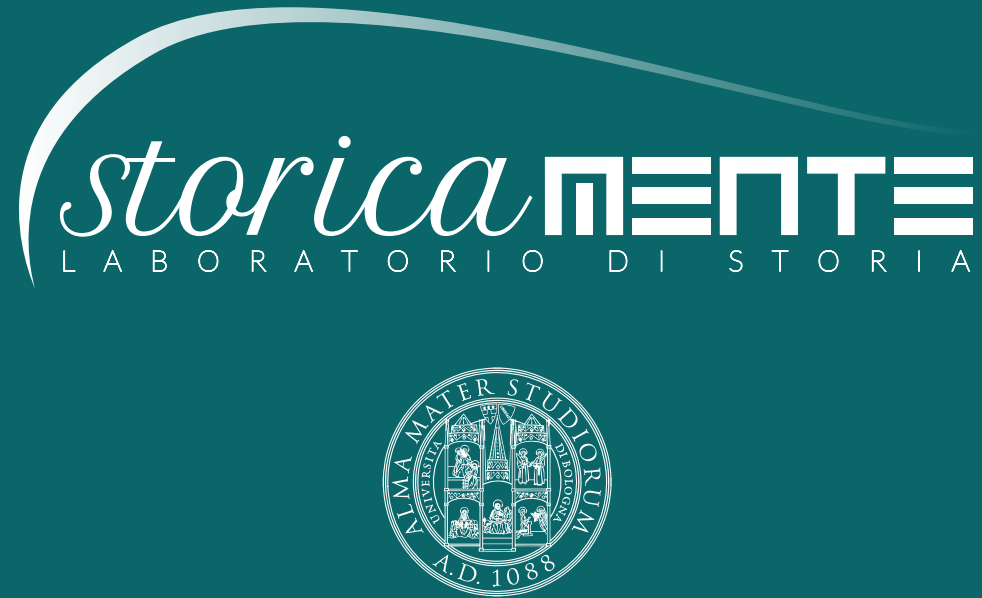

ALMA MATER STUDIORUM

Università di Bologna

Dipartimento di Storia Culture Civiltà

BIBLIO

TECA 


\section{STORICAMENTE.ORG}

\section{Laboratorio di Storia}

Maria Pia Casalena

Maria Antonella Cocchiara, Donne Politica Istituzioni e Società. Temi e questioni di genere, Roma, Aracne, 2016, 581 pp.

Numero 13 - 2017

ISSN: 1825-411X

Art. 14

pp. 1-3

DOI: $10.12977 /$ stor666

Editore: BraDypUS

Data di pubblicazione: 17/07/2017

Sezione: Biblioteca 


\title{
Maria Antonella Cocchiara, Donne Politica Istituzioni e Società. Temi e questioni di genere
}

\author{
MARIA PIA CASALENA
}

Univ. Bologna, Dipartimento di Storia Culture Civiltà

Maria Antonella Cocchiara, Donne Politica Istituzioni e Società. Temi e questioni di genere, Roma, Aracne, 2016, 581 pp.

I saggi raccolti in questo volume - uscito postumo - testimoniano dell'impegno portato avanti dall'a. attorno a temi quali la storia della cittadinanza e la condizione femminile nell'Italia e nel mondo di inizio XXI secolo. Si tratta di un torno d'anni nei quali, a dispetto delle conquiste che si potrebbero definire - seguendo la falsariga delle definizioni utilizzate nei saggi - «formali» (giuridiche, mediatiche, ecc.) non sono in verità corrisposti avanzamenti «sostanziali» per quanto concerne il mutamento culturale, l'affermazione diffusa del femminismo (tanto nella linea della «uguaglianza della differenza» quanto come "pensiero della differenza»). Almeno, come viene qui ribadito, tali progressi non si sono verificati nel campo, ben noto alla studiosa, della rappresentanza e più generalmente del "potere politico».

Con questo volume si inverano due aspirazioni della comunità delle studiose di Gender Studies. In prima istanza, il dialogo tra storia "generale» (politica, ma anche sociale, economica, culturale) delle donne e un 
settore qui definito «di nicchia» e tuttavia riottoso quale la Storia delle istituzioni politiche. In secondo luogo, abbiamo la dimostrazione che l'attività di ricerca scientifica e l'attività didattico-istituzionale appaiono, nel mondo accademico, strettamente intrecciate e anzi indissolubili per quante/i vogliano operare quello stesso mutamento culturale di cui si lamenta l'assenza. Infatti Cocchiara ha affiancato a saggi - inediti o di difficile reperimento - condotti sulla storia giuridica e istituzionale delle italiane, altri interventi scaturiti specialmente dal coinvolgimento diretto nei territori ancora da consolidare dei Comitati Unici di Garanzia e dell'impegno contro la violenza di genere. Nel complesso, così come la dimensione storico-scientifica emerge a più riprese anche negli interventi più "pratici», la dimensione dell'engagement personale e intellettuale non manca di animare le selezioni, le argomentazioni, la stessa scelta delle fonti e della bibliografia di riferimento allorché si tratta della tortuosa vicenda del rapporto tra le italiane e i diritti a partire dal Risorgimento.

Impossibile in questa sede dar conto di tutti gli spunti, le questioni, le conclusioni - più spesso amare - che si traggono dalla lettura dei testi della prima parte, raccolti appunto nella sezione storico-giuridica. Nell'introduzione al volume l'a. dichiara recisamente che l'incremento numerico delle donne nel Parlamento e nei dicasteri non è affatto corrisposto ad un progresso della cittadinanza «sostanziale» della popolazione femminile italiana - né ha dato luogo ad una rappresentanza connotata da una autentica soggettività e solidarietà di genere. Dunque, percorrendo i contributi sulla storia del suffragio, del lavoro, del diritto di famiglia, delle attiviste siciliane del secondo dopoguerra, non si può non dare ragione alla tesi di Michele Ainis - ripetutamente citata da Cocchiara - secondo la quale le conquiste legislative (tardive, parziali, contraddittorie, lacunose in parecchi casi) non abbiano avuto, nel caso delle italiane (come peraltro in altri paesi), ricadute pratiche commensurabili nella società, nel mondo del lavoro, nella sfera politica e neanche nella società familiare (utilissimo a proposito il saggio 
sulla storia della violenza coniugale tra eredità romanistica e mentalità tradizionaliste). I movimenti femministi - da Mozzoni all'UDI, da Kuliscioff al CIF, dalle murriane e dalle "pragmatiche» fino alla svolta successiva al Sessantotto - sembrano tanto numerosi e consapevoli quanto poco radicati, sconfitti in partenza dalla mentalità positivista prima, da un secolare sistema "andro-gerontocratico» e dal pluridecennale retaggio fascista poi, indeboliti (quando non frammentati) da una ingerenza vaticana particolarmente pesante in occasione del dibattito costituente sulla famiglia, nonché da tanti altri fattori ostativi. Conquiste ve ne sono state, questo l'a. lo ribadisce con forza mentre ripercorre la parabola che dall'affermazione delle pari opportunità ha condotto all'aporia irrisolta delle quote rosa in politica. Tuttavia solo la conoscenza storica può risvegliare in giovani (ambosessi) scarsamente consapevoli la piena conoscenza di quanto ancora - nel «sostanziale» oltre che nel «formale» - vi sia da fare. E tale conoscenza viene attinta tanto dalle colleghe dei settori politico-sociali quanto anche da nomi come Rossi-Doria, Salvati o Fiume, ciò̀ dalle pioniere della ricerca storica sui problemi della cittadinanza femminile. C'è quindi tanto da fare in un'Italia che, coi governi più recenti - questa l'«occasione» polemica della raccolta, esplicitamente dichiarata da Cocchiara -, ha risalito numerose posizioni nella scomoda classifica della femminilizzazione della decisione politi$\mathrm{ca}$, esponendo tuttavia molto più spesso personaggi allineati e di facciata che non testimoni e portavoce autentiche di una soggettività che sarebbe essenziale non solo a metà della popolazione, ma alla politica nazionale e internazionale globalmente intesa. 